Meta

Journal des traducteurs

Translators' Journal

\title{
ROSS: Semantic Dictionary for Text Understanding and Summarization
}

\section{Nina N. Leontyeva}

Volume 40, numéro 1, mars 1995

URI : https://id.erudit.org/iderudit/003464ar

DOI : https://doi.org/10.7202/003464ar

Aller au sommaire du numéro

\section{Éditeur(s)}

Les Presses de l'Université de Montréal

ISSN

0026-0452 (imprimé)

1492-1421 (numérique)

Découvrir la revue

Citer cette note

Leontyeva, N. N. (1995). ROSS: Semantic Dictionary for Text Understanding and Summarization. Meta, 40(1), 178-185. https://doi.org/10.7202/003464ar
Résumé de l'article

Le dictionnaire sémantique russe à usage général (ROSS) est un outil pour I'analyse sémantique et informationnelle de tout texte russe cohérent. La structure de ROSS reflète la philosophie et les niveaux de représentation adoptés par le système de comprehension de texte POLIText actuellement en cours d'implementation à l'Institut des États-Unis et du Canada. 


\section{ROSS: SEMANTIC DICTIONARY FOR TEXT UNDERSTANDING \\ AND SUMMARIZATION*}

\section{RESUME}

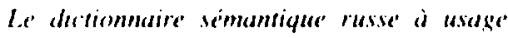
général (ROSS) ast un ousil pour l'amalyse sémunnque et informastonnelle de mot levte rasse coherem las structure de ROSS reflete la philo

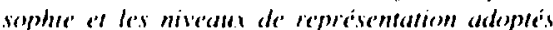

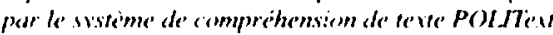

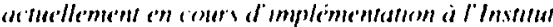

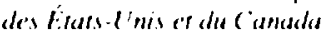

\section{THE PIIRPOSF OF ROSS}

The Russian general-purpose semantic diclionary. ROSS, is a t(x)l for the semantic (both linguistic and informational, analysis of tex(s. The rich semantic information contained in the dictionary makes possible lecal (withın one phrise) semantic interpretation as well as the emantic analysis of coherent texts. Some sones of the dictionary provide the possibility of logico-situational analysis of a kext and a link to different domains.

The present version of ROSS is designed. firstly. to construct a base of textual facts (BTF) for a given collection of Russian lexts. The vexithulary of the the present version is batsed on communications about political events. The procedure proposed for BTH construction (1eontyeva 1992) enables any user 10 form an individual BTF ordering the desired degree of compression of the initial content. Secondly, the dictionary is 10 ensure muli-language, above all, Russian-to-linglish. knowledge balsed machine tramslation (KBMT) (see Johnson. Kang and Ixes Tombe 1985: Nirenburg 1989: Papagaaij 1986).

Though similar in many aspects to dictomaries with rich semantic data belonging to some MT 
systems (e g. Papagaaij ef al. 1986, Шلаляпинна 1974: Anpecar et al. 1992), ROSS differs from them essentially. ROSS as well as the main semantic dictionary (Jeortibeba HH ef al 1979) of the FRAP syslem (French-to-Russian automatic Iranslation implemented by 1985. (see Marhine Translartern and Applied Linguisfics 1987 and Hutchins 19861 implies a clear-cut distunction between the syntactic and semantic levels of text representation. Moreover. semantics itself is split into several sublevels. making possible very important information processes in a system of text understanding, such as tuning to the required domain. shifting the focus of interest. compressing and varying of the initial text content, etc.

The nature of semantic information in ROSS will be clearer if we outline the stages in the understanding of natural language texts (NLT), that is, the linguistic model underlying toth text analysis and ROSS as its instrument, which are being implemented in the system POI.IText.

\section{STAGES OF THE NLT UNDERSTANDING PROCESS}

For us, text understanding is very closely related to information analysis. The text understanding process is a sequence of operations that generate information with the desired degree of completeness from any given NLT for any given reader. We distinguish three major steps in this process (Leontyeva 1987):

1) a complete linguistic analysis of all NLT sentences, which includes all traditional levels of parsing and produces a sequence of representations for a given NLT - graphematic, morphological, syntactic and local semantic: Graphr. MorphR. SynR, local SemR, the last one having the form of initial semantic space (SemSpace). Similarly, any intermediate. not final syntactic structure may be called "syntactic space" (SynSpace); we apply this notion also for the whole text when it is partially processed by the syntactic analyser (e.g. only noun and verb phrases)

This step (or stage) may be characterised as "local understanding" of a text:

2) construction of an internal textual representation in terms of textual fact (TF) constituents. The construction of TF units is a generation process which begins within analysis. This second step of text analy. sis is "global understanding": one TF may be gathered from the lexical material of the whole text and represented in the global TF structure with the required degree of compression:

3) developping the TF structure into information structure in terms of the given domain and/or according to the user's request or information interest. The global TF structure must be matched against the dictionary of the chosen domain objects, i.e. "rewritten" in terms familiar to the given addressee of information (that is, "matched" against and tuned to the lexicon of the user). II may be focused around the required notions as well. This third stage produces units valid. or informative, for the reader or any other perceiving intellectual system. We therefore call it "relative understanding".

We do not consider the process of translation into another NLT as one more level of text understanding. The final representation of every above level could be translated into some another NLT or even into units of another semiotic system (e.g. the "language of actions"), - the results of translation may rather show what kind of understanding has excured: local, global or specialized (relative).

In planning translation component for our POLIText system we have chosen the second and third kinds of understanding. We proceed from the assumption that they involve less computation than every-delail-of-the-text understanding, not to mention the fact that the user needs not the finest information from the text, but only essence ( $f$. Shank's approach). So, we aim at building the TF structure or the preceding structure -- situational representation (SitR) and at translating them into another natural language. In this context, the task of transiation is similar to text generation system tasks, though more complex.

\section{THE STRUCTURE OF ROSS}

The structure of ROSS is hierarchical: the lower level comprises fields that assume concrete values. The higher level is represented by zones, i.e. names of groups of fields. The present version of the dictionary contains 10 zones, comprising over 50 fields. The list of zones is given below.

(Note that all the names of tones and fields, as well as the examples below, are in Russian in the original version of the ROSS dictionary. We have converted most of them into English to make the paper accessible to a wider scientific audience.)

GEN: General information on the word (C) to be described;

MORPH: Morphological characteristics, including the word-forming potential:

SYN: Syntactic properties of the word C (syntac(ic class, specific syntactic structures, complements);

L.EX: Lexical combinability, cliches, idioms, lexical functions:

SEM: Semantic characteristics of $C$. semantic valencies, hypothetic realisation of valencies within one phrase and through the whole text. Actant lexical functions and non-standard questions to actants. Necessary corrections of and other semantic operations on the $C$-containing primary semantic representation;

THES: Thesauric links of $C$ as a concept within a certain domain, C-containing terms. explicit definitions of concepts, encyclopedic functions:

SIT: Sincture of situations related to the semantics of $C$ and relevant to the given subject area, relationships among situatıons, with particular emphasis on relations of temporal precedence ("before") and consequence ("after"); 
PRAGM: Pragmatics of the situation in the domain: events. inferences. presuppositions. evaluation of the event or its parts:

EQIIIV: Equivalents of the initial lexeme cand terms within the entry) into other languages (with selection criteria):

COMM: Comments of the lexicologist ( + name)

Some cones and their fields need more detailed consideration especially when they are non-trivial or different from standard patterns.

Zone (GEN. Fields: TITI.F, TYPF, (AT, NO, REF, OTHER, II.I.

TITLE - name / title of the entry, $t$. ${ }^{\text {. any lexical }}$ unit which can be described semantically. In the present version of ROSS, the entries are represented by one-word lexical units (TYPE $=$ LEX).

- CAT - xemantic category of the lexeme related to the way it is represented in the vemantic notation. This field can acquire the following values:

- I.BL - word-label. 1akes up position $A$ or $B$ in the semantic relation $R(A, B)$. The words of this category have the maximal initial informational weight. This is the largest. open and mobile class that must cover the lexical nucleus of the chosen domain. With ROSS tuned to different domains, the grealest changes in the composition, system of meanings, system of references to the vecabularies of the subject area excur in the L.BL, calcgorie. There are two sub. categories in category $\mathrm{L} . \mathrm{BL}: \mathrm{SIT}$ and OBJ (SITuation. c.s. nar. discessiont. and (OBJect, C.g. Pressdem. book. hill). Those words-labels which can not be specified as SIT or OBJ yield the L.BL. symbol (d.s. problem). The field CAT provides the highest level of classification: further semantic differentiation of words-labels is derived from fields SEMF (semantic features) and VAL (valency) of the rone SE:M

- REL. - relations (or semantic relations, SemRel) (xcupy position $R$ in the formula $R(A, B)$. These words have lower, as compared to I.BI., initual informational weight since they demote links (relations) between units of other categories. In the majority of cases, REL, is ascrited to auxiliary parts of speech (prepositions, conjunctions, punctuation marks). Nouns that coincide with the name of some semantic relition (cause, parl) fall into the category of aspect words: they become relations only at the next step of analysis

- ASP - - aspect words. They have a common structural feature, tes. the way they are represented in the semantic notation: the lexeme defines the name of the semantic relation and fills in its first place in the formula $R(A, B)$ : PARAMETER(size,B): PART OF(member, B).

Aspect words are non-homogeneous both grammatically (nouns, adjectives, verbs, adverbs) and semantically, and they can be specified in SEMF and VAL fields of SEM rone (see below) hy more specific semantic relations: STACEE(A.B), MODALITY(A.B). NAME(A,B). FINCTION (A,B), INDENTIFIER(A,B), and others. ASP words have lower informational weight than the aboive.
OPER - words-operalors whose individual hehaviour is described by algorithms of Iransformation of a part of semantic representation (SemR): each operator has its own sphere of action and leads to a tairly complicated transformation of already constructed part of SemR. This catcegory comproses pro-

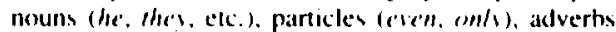

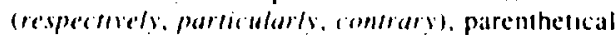
words (in parficular, medemalls), yuantors feleres. all). etc. These units operate on SemSpace structure (at the sage of semantic analyss proper) when there alleady exist unts that can become arguments of the semantic relations that are introduced

The informational weight of these lexemes given in the dictionary is monimal, but the transformations they start may change the informational weight of term $B$ in the semantic formula.

- ELSE: - a quasi-calegory for doubiful and unclear cases.

No) the ficld to indicate the number of meanings (in brackels) and the ordinal number of the given one: $\operatorname{se}(1 / 2)$

RII: reference to a word or its individual cones and fields (in ROSS ) with similar information: REF is used for unification of information and effortsaving input, e. TITLE: - he; NO - 2(2); REF keyl (MORPH. SYN), which means that hele (e.g Then is the ket to the woluriom of ente problem) has the same MORPH and SYN Aone as keyl (e.g. Open the door nith a Re'v)

OTHI:R ather meanings. The field ondicales (in a free form) other meanings (not yet described in the dictionary) to provide a contrasting example or to prepare the ground for further description in the dictionary.

Il.l. Illustrations: an example of the most typical context for the given meaning of the word. The word itself is substituted by - Illustrations can be also given in the fiekd II.L.1-7 of the SEM rone with the emphasis on each of the satisfied valencies.

\section{Zone MORPH. Fields: MC:., (HANGE, DFRIV}

MCL. morphological class: each class has its own morpholegical characteristess. For example, for verbs: CASP - grammatical aspect of the verb: (perfect, imperfect, perf / impert, and so on).

TRANSIT = transitivity $("++"$ and $" \cdot-")$.

REFLEEX = reflexivity ("“+" and "."”).

CHANGE availability of the paradigm $("+, " . . ")$.

DERIV .- morphological derivation. The field denotes the ways to form other parts of speech from the given TITL.F, as well as reflexive forms, comparative and superlative degrees of eomparison, negative forms. For verhs, the field indicates:

the way lo form the aspect pair, es

TITL.E = petllalb: DERIV = PERF $(-3+m$ )

- the way to derive the passive form, ce.

TITLI: = pe'llatr; DERIV = PASSIV (anal. ( analytically and with the help of the positix. 
- potential word formation through prefixes, e.g TITLE = делать: DERIV = PREF $\left(\mathrm{ma}^{\sim}\right) .\left(3 \mathrm{a}^{\sim}\right)$.

More complex forms of derivation are described in zone LEX and field ALF of zone SEM.

\section{Zone SYN. Fields: SynCl, ROLE, COMPL, CONSTR} (verb)...

SynCl - syntactic class: NOUN (noun), VERB

ROLE -- role in the phrase: NP (noun phrase), VP (verb phrase), ATTR - attribute, etc.

COMPL - complement, "strong governmeni". in the form of a) a pair "preposition and the symbol of the noun's case", P.g. "B + Acc"; b) the symbol of the noun's case ( 6 cases in Russian); $c$ ) the name of syntactic class.

CONSTR - prediction of non-Irivial syntactic constructions containing the title word as the key one.

Zone LEX. Fields: P-len, P-right, COLLOC, LF phrases.

P-left, P-right - left and right parts of fixed

TITLE = power: P.left $=$ under $\sim$ :

COLLOC - different non-free collocations:

TITLE = peace: COLLOC = the cause of peace: peace champors:

$\mathrm{LF}=$ lexical functions (according to the "meaning-text" theory, see Mel'cuk 1988).

TITLE = depury; $\mathrm{LF}=$ Oper, have a deputy (of their own); Oper: he a deputv: Caus, Oper: elect a - SI elector: Fact: justify the trust of electorate.

Zone SEM. Fields: SEMF, VAL1-7, SEMF1-7, ALF1-7, QUEST1-7, SYN1-7, MORPH1-7, ILI.1 -7, POS, INCOMP, COMPAT, ADD,

CORR, RESTOR, OBLIG, MODIF,

TRANSF(SEMF), TRANSF(VAL)

SEMF - semantic feature, or characteristic (see the list below p. 1831 .

VAL - - a set of valencies of the word (up to 7 ): candidates for filling in the slots of valencies are introduced by symbol Ai $(j=1,2 \ldots 7)$. The form of notation in VAL. field: R,Ai,C or R.C.Ai. TITLE = message: $V A L=$ agent $A 1, C:$ addressee, $A 2, C$ : topic, A3,C: content,A4,C

SEMFi - semantic feature of each possible actant of each valency

ALFi - actant lexical functions:

TI1LE = elect: ALFI = elector. electorate.

QUESTi - non-trivial question to each actant.

SYNi - syntactic characteristics required of actants; syntactic relations between the actant and $C$.

MORPHi - morphological characteristics required of each actant.

ILLi - illustrations for each valency.

POS --. interrelated positions of $C$ and its actants in the phrase:

TITLE = delegate: MORPHI $=1$, from $+\mathrm{N} ;$ MORPH 3 $=1$ of + N: POS $=<$ MORPH $3.1<$ MORPH 1.1 (delegate to the (ingress from the state of Michigan).
INCOMP - incompatibility of morphological characteristics of actants; written in the form of conjunction of MORPH's.

COMPAT - compatibility of MORPH's and/or SEMF's; written in the form: inference, slash. condition, $e .8$

TITLE $=$ compensation: VAL $=$ agent.A1.C: ob.A2.C: addressec, A.3.C

MORPHI $=1$. poss.pronoun; 2. adj: 3. of $+N ; 4$. by + N:

MORPH2 $=1$. of $+N: 2$. for $+N$ :

INCOMP $=$ MORPHI .3 and MORPH2.I

COMPAT = MORPH2.1/MORPH 1.4 (lf there is

MORPH1.4, then there is also MORPH2.1), e.g. $\mathrm{com}$. pensation of damages: compensation of damages by an insurance company: compensation of the insur ance company was too litule.

ADD - additional semantic relations among the actants:

TITLE = compensation: $\mathrm{ADD}=1$. passive actant,A2,A3; e.g compensation to NN(A2) for the damage (A3).

CORR - the rules of correction of the valency structure written in the form: initial SemRel, $\rightarrow$ (symbol of transition), resulting SemRel, / condition: c. $g$.

TITLE = ruin: $\mathrm{VAL}=$ agent, A I.C; passive actant,A2,C;

CORR = agent,Al,C, $\rightarrow$, cause, $\mathrm{Al}, \mathrm{C} / \mathrm{SEMF} 1$ animated; $e g$. The flood $(\mathrm{A} I)$ has ruined the village (A2).

RESTOR - the rules for reconstructing a member of the valent stnucture, in particular, the subject of action expressed by infinitive:

TITLE = order: VAL = agent,AI,C: content,A2,C: addressee. A.3.C

MORPH2 $=1$. INF; RESTOR = agent, A3,A2/

SEMF2 = action; e.g. The commander $(A 1)$ ordered

his soldiers( A.3) to remuin(A2) seated.

OBLIG - obligatory realisation of a valency TITLE $=$ order $;$ OBLIC $\mathbf{i}=2$.

TRANSF(SEMF) - (ransformation of semantic characteristic, tee. regular change of semantic charac teristic of $\mathrm{C}$ under certain conditions (presence of certain grammatical features, or presence of a certain context), t'.s

TITLE = stress; SEMF = phenomenon: TRANSF (SEMF) = parameter $/$ value.Al.C: (of Stress is a physical phenomenon vs The stress nas equal to $(.5 j(k g)$

TITLE = audience; SEMF = receptacle: TRANSF $($ SEMF $)=$ anim, assemblage

TRANSF(VAL) = transformation of valencies, written in the form: Ri, Rj. which means that a strong syntactical valency which serves to express $R i$ becomes a means of expression for $R j$. while $R i$ becomes completely devoid of a strong syntactic expression, $e g$

TITLE = publish: VAL = agent,AI,C: ob,A2,C: Ioc, A., C: MODIF = agent, loc (The new:spaper(Al) 
has published an article( $\mathrm{A} 2) \rightarrow \operatorname{Some} b o d y(\mathrm{~A} 1)$ published an article(A2) in the newspaper(A.3)):

\section{Zone THES. Fields: DOM, TERM, HIGH, ENCYC, VAR, ASSOC, EXPI.IC}

IOM - dom:in. or subject area, one or several of the following: conomics, politics, jurisprudence. military problems, general vocuabulary. In brackets, we indicate the informational weight of the word (5 - for the most informative words. 1 - for terms indifferent to given DOMain. ('.s.

TITLE = rectirom: DOM = politics(5).

TERM - (-containing terms which must be then incorporated to ISC RAN databases, o e

TITLE = comention: TH:RM = ciene'va Humanitarian Convernion.

HIGH - generic concepe for $C$.

TITLE: $=$ component: HICHH $=$ part

TITLE $=$ hill; HIGH $=$ der $u$ umem:

TITLE: = depurv: HIC socion.

ENCYC - encyclopaede functions (Anti. Mult. Pars, Param, Sing):

TITLE = senator; ENCYC = Mult: Se'nate:

TITLE $=$ depulv: ENCYC $=$ Mult: Purliame'm

Congress. Supreme Soriert:

TITLE: = morfit: ENC'YC' $=$ Anti: damuse' loss:

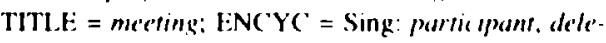
galle

VAR - variants of c (of different degrees of closeness):

TITLE $=$ compensafion: VAR $=$ contribution. insur . ancer, reimburseme'm, inde'mmificanion.

ASSOC = other associated concepts

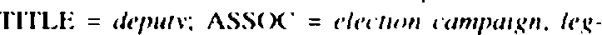

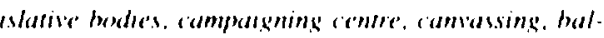

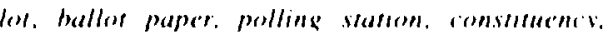

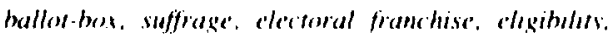
polling district, glasmost, perestroika

EXPLIC - definition or explacit description for words with informational weight 4 and 5 : can be formulated in terms of SIT or taken from an encyclopaedic dictionary.

\section{Zone SIT. Fields: MSit, ESit (KSI-n), \\ PREC C.I), POST}

MSit - main situation, designated as comb.C. $|A|$. where $(A)$ means the sel of actants, and comb indicates its compatibility with $C:$ this notation signifies that the main situation is represented by multi-term predicale $($.

FSit - description of elementary stuations in the form of a set of semantic relations $R, A, B, c . g$ TITLE: = evere: VAL = agent,Al,C; oh.A2, $:$ end-point, $A .3, C$.
ESit $=1$. belongs-to, A2,A1 $/$ SEMF1 = organisation: 2. loc.A 3,A2/SEMFI = space, state: 3. helongs10.A2.A.3: 4. IKC.A3,A2.

Further on, elementary situations are referred to as ES1, ESS2, etc.

PRE(CEI) elementary situatoon preceding MSit.

POST -- elementary situation following MSit, e.g. TITI.F = evpert: PRECED = ESI\&ES2: POST ES3\&ES4.

\section{Zone PRAGM. Fields: EVENT, CONCL,} PRESUP, EVAL, L.OG;

EVENT - event (main situation denoted by the word and/or one of its actants with the greatest informational weight which may be a nucleus of some event in the indicated domain ). $c$ a. EVENT $=A 3$.

CONCl. standard inference in the form of a production rule: II (SITI), then (SIT2): if (SIT2), then NON (SIT3)

PRESUP - presupposition (names of situations already introduced in some field, or being formulated by the linguist, which are indispensible for $C$ to be (rue).

EVAl, - evaluation $1 "+", " . ", "() "$, or " "?", the latter signifies that the evaluation depends upon certain conditions). ce.g.

TITLE $=$ "yom $:$ EVINT = MSit: EVAL $=? /(2)($ is inherited from $\mathrm{A} 2$ ).

I.OK more complicated situations that charac. kerise the logics of the events. formulated in terms of SIT and production rules.

\section{Zone HQUIV. Fields: F.NG, FR}

INC; Inglash equivalents with selection conditions.

F R - French equivalents (not indicated in the present versions.

\section{Zone COMM. Fields: COM. NAMF}

COM - commentary in a free form.

NAME: name or any other designation of the

lexicologist.

\section{SOME REMARKS ON THE SEMANTIC COMPONENT USING ROSS}

The semantic component (SemComp) is responsible, first of all, for the semantic interpretation of all the units of the initial ext (or of its syntactic structure. even incomplete). In our system. Sem(omp receives syntactic space (SynSpace) as a source and makes possible the interpretation of those nexdes and relations of SynSpace on which information is available in one of the semantic dictunaries (ROSSI, ROSS2 ... ROSSn). Secondly, SemC omp must ensure the matching of textual units to and their compatibilit) with the unts of the chosen demain and/or knowledge representation (KnowR). Thirdly, It dow the same in regard of the uniss of information representation: it is Sem (omp that is responsuble for correspondence belween two languages the KnowR of the yystem and the KnowR of the user. 
The initial semantic structure. semantic space (SemSpace), is a result of a local (within the sentence houndaries) semantic interpretation of the initial text in terms of binary relations only, relations not associated with a particular notional sphere or domain. It may be accompanied by the structural decomposition of some lexemes which have valency structure, or by combining some simple nodes of SynSpace into one complex term. all operations being guided by ROSS and the thesaurus of political terms.

From the structural point of view. SemSpace is a sequence of formulas meeting definite rules of semantic grammar, - these are the now well-known semantic triads of the form $R(A . B)$. where " $A$ " is a dependent notion, " $B$ " is a chief notion, " $R$ " is a semantic relation between them. We have used this form of semantic representation since 196.5 . not only for verbs as is in frame-based languages, but for all the lexical material of any text, be it Russian. French or any other.

Here are some examples of semantic features and semantic relations.

\section{SEMANTIC FEATURES (fragment)}

ARTEFACT - man-made object: table, weapon. spurmik:

ABSTRACT CONC. -- theory, system:

SUBSTANCE - sand, clay, uranium;

PERCEPTION - hear, sec, observe:

HARM - anything connected with life hazards: war, threat, damage;

STATE - rowntry, USSR:

MOTION - so, carri, flow, move, boat, train, car:

CHANGE - increase, augmentarion:

INTELLIGENCE - opinion, lecture:

SPACE - river, platform, region:

SEMANTIC RELATIONS (fragment)

AUTHOR, A, B - poem(B) by Kav(A);

ADDRESSEE,A,B - 0 send(B) smith w the press(A);

ASPECT.A,B - $\operatorname{sor}(B)$ by size( $A)$;

TIME.A.B - nent(B) in /99/(A):

VALUE,A,B - velow 1 (B) of $100 \mathrm{~km}$ per hour $\mathrm{A})$ :

IDENTIFIC ATOR.A.B - Presidenn B) Gorbuche'(A);

NAME,A.B - IBM(A) Company $(B)$;

SOURCE,A,B - $\operatorname{laser}(A) \operatorname{rav}(B)$ :

QUANTOR,A.B - each(A) party(B):

MODALITY,A,B - mus/(A) rome(B):

The Semantic Grammar is defined by two axes:

- syntagmatics, that is rules of combining words into formulas. Ex.: REASON,A.B. where A (and also B) = SIT or a whole semantic formula: NAME,A,B , where $A=\operatorname{NAME}(A, ?), B=$ any;

- paradigmatics, that is rules of substitution for terms and relations. Ex.: IDENTIFICATOR,A,B can be specified as (that is, substituted in the course of semantic analysis by) NAME.A.B or SYMBOL.A.B. TIME,A,B can be specified by two formulas: STARTIN(; POINT(?,B) and FINAL. POINT(?,B), etc. There exist more complex relations between semantic units (relations, formulas and other units).
As for features, they are also partially hierarchizied, ex: SITUATION > PREDICATE > PRO). CESS > ACTION. This Grammar is used for compression and other transformations over the SemSpace.

SemSpace is a homogeneous structure. All transformations within it aim at the construction of more "intelligent" units, called situations (Sits) and "textual facts" (TFs). Both are multiplace predicales. The former are buitt on the basis of dictionary descriptions of words and the lexical material of the text, that is they are "local" semantic units. The latter must be "generated" on the basis of the quality of Sits constructed in conformity with rules of global semantic analysis, and the quality of coherent text structure. $A$ TF and its actants are calculated as notions with maximum informational weight (maximally meaningful) for the given text and/or the given thesaurus, domain and pragmatic orientation. (See in Leontyeva 1992 the first attempt to formulate some rules of similar pragmatic analysis.)

Example of a textual fact:

TF1 = COUP D'ETAT $(1,2,3,4,5)$

lexical variant: setzure of powe'

1. Agent = the USSR State Emergency Committee (SEC)

Variant $=$ the Soviet leadership

Identification = G. Yanayev, V.Pavlov, O.Baklanov,

B.Pugo, V.Starodubtsev, A.Tisyakov, V.Kruchkov,

D. Yarov

2. Counter-agent $=$ [former power, President Gorbachev]

3. Cause $=$ destabilisation of political and economic situation in the USSR: Sit

4. Goal = 10 overcome economic and political crisis in the USSR: Sit

5. Time: Starting point $=$ August 19,1491 : Sit

The base of textual facts BTF for a given corpus of texts will be a condensed. secondary structure, result of comparison, generalization and generation of new units which can be translated into superficial forms other than the initial ones, as in the case of knowledge based machine translation.

Text generation (TG) from BTF can yield new lexts, such as English or French summaries of initial Russian texts. Some of the existing multi-language TG projects and systems lsee Mckeown 1985: Kittredge (t al. 1991) allows us to look forward to valid translations from BTFs.

\section{IMPLEMENTATION AND PERSPECTIVES}

Some components of local linguistic analysis in the POLIText system. particularly graphematical. morphological and, in part. syntactic (noun phrases. verb phrases) ones, have already been implemented. At present, domain-oriented analysis is being created. matching every noun phrase against the thesaurus of political terms and thus constructing nodes which 
obtain the denotation status in a future semantic structure.

The next step is the semantic interpretation of all other nodes and relations based upon the ROSS dictionary. The prototype of this moklule and of the domain-oriented module were programmed by $\mathrm{N}$. L.ucashevich.

The creation of the Russian semantic dictionary is the most labour-consuming part of the project. This work is done by a team of five lexicologists of the Moscow State linguistic I Iniversily IM. Shatalova d' al.). ROSS has been entered into IBM P(' by means of a special scenario in an online mode (man-machine dialogue). A special dala base management system. derived from the ('atrion Databises, has heen evolved by $B$. Dobroff, for more flexible storing, upxlating and development of ROSS, including access from applied programs.

\section{CONCLUSION}

When computer systems faced the severe necessity to provide the description of the semantic component. the traditonal linguistics fell short of suitable semantic theories. Meanwhile no serious systematic text processing would te possible without a full employment of the lexicon as a whole. The most vul. nerable area proved to be that of interaction with data and knowledge bases. Usually this problem would to solved by computer scientists, albeit scholars in the field of detailed linguistic analysis made a successtul altempt to "crush through" to the KnowR (Anpecsu ot al. 1942; Boguslavshij and Tsinman $|(9)|$ ).

A full picture of computational semantics was proposed in Pustejovsky 1402. the central idea being that "word meaning is highly structured, and not simply a set of semantic features." "Jwo tasks "a fully compositional semantics for natural language and its interpretation into a knowledge represtoltation moxlel". as well as "the mapping from the lexicon to syntax" - are considered to be the most important.

We see a certain correlation between these ideas and our approach to levels of semantic description.

NinA N. LEONTYYEVA Institute of the USA and Cimald. Russian Acodemy of Solences. Mosions, Russua

\section{Notes}

* The work on the ROSS dictionary was supported by the MacArthur Foundation (a 1994 grant) and the SOROS (Cultural Initiative: a 1994-1905 grantl. I would like lo express my heartell grati. tude wo both toxdies for their support.

\section{RFFERENC:F}

BOGUSLAVSKIJ. I. M. and I. L. TSINMAN (1991): "Semantics in a Linguistic Proxessor". (ompulers and Aruficual lneellagence', n" 3. pp. 3-20.
HOVY, E. F: (1988): "On the Study of Text Planning and Realsalion", AAAI Workshop on Tex Planmng. St. Paul.

IIITCHINS. W. J, (1966): Marhme Tiamslarion: Past. Present. Future. New York, 382 p.

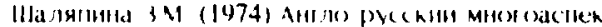

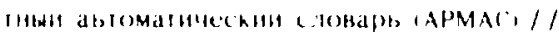

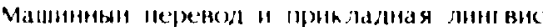

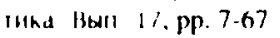

AाIме

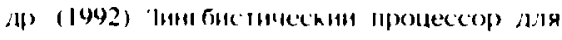

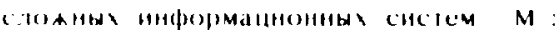
Havia, $250 \mathrm{p}$.

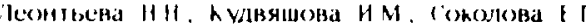

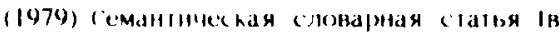

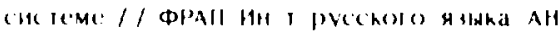
IC(P. BINI1, $64 \mathrm{p}$.

JOHNSON, R., KING, M. and L. DES TOMBE (1985): "EUROTRA: a Multilingual System Under Development". Computamonal tinguistics, Vol. $11, n \cdot 2 \cdot 3$.

KITTREDX ik:, Richard, KORELSKY, Tanya and Owen RAMBOW (1991): "On the Need for Domain Communcation Knowledge". Computalional Imlelligener. Vol. 7. n" 4. December.

I.FONTYIVA. Nina N. (1987): "Stages of Information Amalysis of Natural Language Texts". Im Formm Inf. and Drocum. Vol. 12. n"4.

I.IOONTYEVA. Nina N. (1992): "Textual Facts as Inits of ('ohereme Texi Semantic Analysis". Internatumal Workshop) on the Meanme-Tex Theors, Karen Haenell and Leo Wanner (Fds.). 1)armstadl.

Machere Tramslatron and Applied Linguistics Problenss Related io the Delelepment of Ausomatis Translation Sisfems (1987): Issue 271. Moscow's Maurice Thore, State Instlute of Foreign languages.

MCKFowN, Kathleen R. (1985): "Discourse Strategies for Cienerating Natural-1 anguage Text”. Artifical Intelline'nce, 27.

MEI.C'UK. Igor A. (I988): "Paraphrase et lexique dans la théoric linguntique Sens-Texte". Cahers de levicologie, 52-1, pp. 5-50, 53-2, pp. 5-5.3.

NIRIENBUR(;. S. 1 1989): "Knowlege-based Machine Translation", Machme Translamon, 4-1

PAPACiAAlJ, B. ( , (1986): "Word Exper Semantics. An Interlingual Koowledge-hased Approach", Dismbuted Language Trams/arion. Toon Witkam (I:d. I. Donrecht, Revernon. $254 \mathrm{p}$.

PAPACIAAIJ. B. ( .. SADLER, V. and A. P. M. WITKAM (1986): "Fxperiments with an MTdirecled Lexical Knowledge Bank". ( OLINC;-8s.

PIISTEJOVSKY, James (1942): "The Generative Iexicon". ('omputarional lingusistics. Vol. 17, n'4.

ROESNE:R, D, (1987): "The (ieneration System of the SI:MSYN Project. Towards a Task-independent Ciencrator for cierman". First Furomeson Workshop' an lampluage (ienceration, Paris. 